\title{
Altering and investigating the surfactant properties of graphene oxide
}

\author{
Harish V. Kumar, ${ }^{\dagger}$ Kevin Y.-S. Huang, ${ }^{\ddagger}$ Shawn P. Ward, ${ }^{\ddagger}$ Douglas H. Adamson ${ }^{\dagger, \ddagger, *}$ \\ ${ }^{\dagger}$ Institute of Materials Science, Polymer Program, University of Connecticut, Storrs, CT 06269 \\ ${ }^{\ddagger}$ Chemistry Department, University of Connecticut, Storrs, CT 06269
}

ABSTRACT: Graphene oxide (GO) is a two-dimensional material with a hydrophobic carbon lattice functionalized with hydrophilic oxygen groups on the edges and basal plane. Its hydrophobic/hydrophilic nature allows it to behave as a surfactant, stabilizing emulsions of oil in water. In the investigation described here, we study GO's emulsifying ability by using it to template the polymerization of polystyrene while tuning the hydrophobicity and degree of oxidation of the GO sheets. SEM, TGA, optical microscopy, acoustic spectroscopy, and digital image analysis are used to characterize the system. The size of the GO templated PS spheres is found to be a function of the amount of GO in the system, with too little GO resulting in PS spheres with no GO shell. Increasing the hydrophobicity of the sheets is shown to lead to the formation of inverse emulsions, while increasing the graphitic character of the GO results in distorted styrene droplets as the GO sheets become more planar and less able to conform to a smoothly curving interface.

Keywords: graphene oxide; 2D surfactant; GO fractionation; emulsion templated polymerization

\section{Introduction}

The oxidation of graphite to form graphite oxide (GO) was first reported over 150 years ago in an attempt to determine the molecular weight of carbon,[1] and more recently has been used to facilitate exfoliation and impart water solubility to graphite for processing.[2,3] Being derived from graphite, GO is composed of two-dimensional sheets of carbon, but with oxygen groups at the edges and in regions of the basal plane. This makes GO amphiphilic, and has led to its use in stabilizing emulsions. [4-7] We have investigated the surfactant qualities of GO by studying the mechanism of emulsion formation, the effect of GO concentration on the size of the dispersed phase droplets, the effect of changing GO's hydrophilic/hydrophobic balance, and by tuning the properties of the GO sheets to alter the morphology of the emulsion droplets. 
Previous studies in which GO has been utilized to stabilize oil-in-water emulsions[8,9] have described the emulsions either as Pickering emulsions or as surfactant-stabilized emulsions. The fact that GO emulsions are described in both ways is a result of the unique two-dimensional morphology of GO that allows it to behave as a molecular or a colloidal surfactant.[10] Examples of these studies include: the work of Thickett, et al., where the identity of the oil phase was shown to play a role in the stability of GO emulsions, showing that nonpolar oil phases were favored,[11] investigations by Guo, et al. where hollow GO spheres in olive oil were created by heating GO stabilized emulsions,[6] and Gudarzi, et al. who demonstrated that GO-stabilized Pickering emulsions could be used as precursors for emulsion polymerization.[9]

Extending the use of GO to emulsion templated polymerizations, several groups have reported the formation of polymer particles,[3-5,12-14] macroporous composites,[15] and magnetic nanoparticles[16] templated by GO emulsions. Yin, et al. suggested that the formation and adsorption of polystyrene oligomers to the surface of GO sheets via $\pi-\pi$ stacking and $\pi$-bonding interactions led to rapid aggregation of the GO at the interface, and that the polymerization occurred via a nucleation and growth mechanism starting from the surface of the GO.[3] Sun, et al. observed that if a conductive polymer such as polyaniline was used in the emulsion, polymer/GO composites with improved electrochemical performance could be produced.[12] Xie, et al. proved that the $\mathrm{pH}$ of the solutions played an important role in the stability of the emulsions, while the size of the GO sheets influenced the morphology of the polymerized particles.[4] The application of small, less than $100 \mathrm{~nm}$, GO sheets has been shown in a series of articles to allow microemulsion polymerization leading to the formation of GO coated spheres with diameters in the $0.5 \mu \mathrm{m}$ range.[17-19]

An important aspect not addressed in previous studies is the tremendous variation found in GO. Different batches of GO can vary widely in terms of overall degree of oxidation[20] and chemical functionality,[21] depending on the method of oxidation and work up. GO is a very polydisperse material in 
terms of the level of oxidation of individual sheets, and every batch of GO has a unique distribution of oxidation.[22] The oxidation process requires the use of harsh conditions, often involving sulfuric acid and potassium permanganate, and produces sheets with a wide range of oxidation levels, with some sheets highly oxidized and others having nearly no oxidation.[23]

In this paper, we examine the effect of surface functional groups, dispersity of oxidized sheets, and the role of GO concentration on the surfactant properties of GO. Using styrene as the oil phase in GO stabilized oil-in-water emulsions, followed by polymerization of the styrene to polystyrene (PS), we show that it is the GO concentration in the emulsion, and not the emulsification process, that determines the size and composition of the product, and by converting the hydrophilic oxygen functionalities to hydrophobic groups we elucidate the importance of the hydrophilic/hydrophobic balance of the GO sheets. Finally, using a fractionation approach recently developed in our lab,[24] we investigate the role that oxidation and sheet stiffness plays in GO's surfactant properties.

\section{Experimental}

Synthesis of Graphene Oxide Graphene oxide was synthesized from natural flake graphite (Ashbury Mills grade 3243, $50 \mu \mathrm{m}$ sheet size) using a modified Hummers' method.[25] $625 \mathrm{~mL}$ of concentrated sulfuric acid and $12.5 \mathrm{~g}$ of sodium nitrate were stirred for 30 minutes prior to the gradual addition of $25 \mathrm{~g}$ of graphite over a 20 minute period. This was followed by the addition of $75 \mathrm{~g}$ of potassium permanganate. After 2 hours, the reaction was quenched with $1.5 \mathrm{~L}$ of water, and $625 \mathrm{~mL}$ of hydrogen peroxide were added to oxidize the remaining manganese salts. For workup, $625 \mathrm{~mL}$ of concentrated hydrochloric acid was added. The resultant GO was centrifuged and filtered prior to being ground in a ball mill to break large chunks into powder. The Raman spectra and XRD of the GO is shown in SI 1.

Emulsification of Water/Styrene Mixture in the Presence of Graphene Oxide GO was added to DI water at concentrations of $0.1,0.4,0.7,1.0,2.0$, and $4.0 \mathrm{mg} / \mathrm{mL}$ of total solution and suspended using a bath sonicator for 15 minutes to disperse the GO sheets. Emulsions were made with water/styrene ra- 
tios of both $70 / 30$ and $30 / 70$ by volume. For the 30/70 v/v styrene/water emulsions, this resulted in samples with the mass of graphite oxide relative to styrene $(\mathrm{mg} / \mathrm{g})$ being: $0.37,1.46,2.56,3.67,7.33$, and 14.65 . For the $70 / 30$ styrene/water emulsions, the mass of graphite oxide relative to styrene (mg/g) was: $0.16,0.63,1.10,1.57,3.14$, and 6.28. In the styrene phase, $9 \mathrm{mg}$ (for 30/70 o/w) and $21 \mathrm{mg}$ (for $70 / 30 \mathrm{o} / \mathrm{w}$ ) of azobisisobutyronitrile (AIBN) was added and $0.375 \mathrm{~mL}$ (for $30 / 70 \mathrm{o} / \mathrm{w}$ ) and $0.875 \mathrm{~mL}$ (for 70/30 o/w) of divinylbenzene (DVB) was added for a 10\% target degree of crosslinking (i.e. 10 mol\% of the monomer oil phase). The mixtures were hand-shaken for $10 \mathrm{~s}$ prior to blending for $30 \mathrm{~s}$ with a Kinematica Brinkmann Polytron Homogenizer (Model PT 10-35). The emulsions were then placed in an oven at $65{ }^{\circ} \mathrm{C}$ overnight to polymerize. After polymerization, the vials were placed in a second oven at $80{ }^{\circ} \mathrm{C}$ overnight to dry. Unless otherwise noted, the conversion of monomer was $\sim 100 \%$.

Effect of Blending Time on GO-Coated Polystyrene Spheres Samples were prepared using a 30/70 (v/v) styrene/water ratio with a $1.0 \mathrm{mg} / \mathrm{mL}$ concentration of $\mathrm{GO}, 9 \mathrm{mg}$ of $\mathrm{AIBN}$, and $0.375 \mathrm{~mL}$ of DVB. The samples were blended for $0.5,2,5$, and 10 minutes, polymerized, and collected as described previously.

\section{Analysis of GO-Coated Spheres Emulsion Sphere Size Analysis}

The emulsion sphere size was determined using a DT-1202 acoustic and electro-acoustic spectrometer from Dispersion Technology Inc. Ultrasound attenuation spectra in the frequency range from 1 to $100 \mathrm{MHz}$ was analyzed with Dispersion Technology software for polydisperse emulsions to obtain the sphere size distribution.[26] The emulsion utilized water as the continuous phase and styrene as the dispersed phase. After the emulsion finished mixing it was allowed to sit for 3 hours before being loaded into the instrument.

Field Emission Scanning Electron Microscopy (FESEM) was performed on a JEOL JSM6445F/Thermo Noran System Six EDXS with an accelerating voltage of $10.0 \mathrm{kV}$ to analyze the surface characteristics of the spheres. Samples were coated with a gold/palladium alloy using a Polaron E5100 
SEM coating unit. Optical microscopy was employed to measure the sphere sizes using a Nikon Diaphot TMD inverted microscope. Images were calibrated using a stage micrometer and sphere sizes determined using ImageJ image processing software. Thermal gravimetric analysis (TGA) was performed on a TA Instruments TGA Q-500. Samples were loaded into platinum TGA pans and heated from $20{ }^{\circ} \mathrm{C}$ to $800{ }^{\circ} \mathrm{C}$ at a rate of $20{ }^{\circ} \mathrm{C}$ per minute in a nitrogen atmosphere.

Silanation of Graphene Oxide and Phase Inversion of Emulsion Three batches of graphene oxide were dispersed in dry THF $(7.5 \mathrm{mg} / \mathrm{mL})$ then bath sonicated for 2 minutes. Trimethylchlorosilyl chloride (TMSCl) was added to each vial with $20 \%, 60 \%$, and $100 \%$ target degrees of silanation. The vials were stirred overnight prior to evaporation of the THF. The silanated GO samples were analyzed using TGA from $20{ }^{\circ} \mathrm{C}$ to $900{ }^{\circ} \mathrm{C}$ at a rate of $20{ }^{\circ} \mathrm{C}$ per minute in air. These silanated GO samples were then used to stabilize styrene/water emulsions for polymerization using the previously described procedure.

Fractionation of Graphene Oxide Fractionation of the GO is described in detail elsewhere.[24] To briefly summarize, $400 \mathrm{mg}$ of GO was dispersed in $50 \mathrm{~mL}$ of DI water, then bath sonicated for 5 minutes. $50 \mathrm{~mL}$ of chloroform was added and the mixture was shaken to emulsify the chloroform. GOstabilized chloroform droplets were allowed to settle to the bottom of the jar and the upper aqueous layer was decanted. The solvents were evaporated and two fractions of GO, GO emulsion and GO water, were recovered. GO-coated polystyrene particles were made and analyzed by the previously described method using these fractionated GO samples.

\section{Results and discussion}

Morphology of PS Spheres with GO Shells The stabilization by GO of a styrene dispersed phase in a water continuous phase, followed by the polymerization of the styrene phase, results in the formation of solid spheres with GO at the surface. In order to visualize the GO coating, the spheres are heated above the decomposition temperature of PS but below the decomposition temperature of GO. TGA of the spheres (Figure SI 2) indicates that at $450{ }^{\circ} \mathrm{C}$ the PS decomposes, leaving the GO shell behind. Fig- 
ure 1 shows FESEM images of the spheres after heating to $450{ }^{\circ} \mathrm{C}$. A distinct core-shell structure is observed, as a portion of the PS is removed, resulting in the core shrinking slightly from the now cracked GO skin. This core-shell morphology differs from previous studies that report either surfaceadsorbed[13] or intercalated composite structures.[14] What we observe are PS spheres defined by a layer of GO that had resided at the water/oil interface.

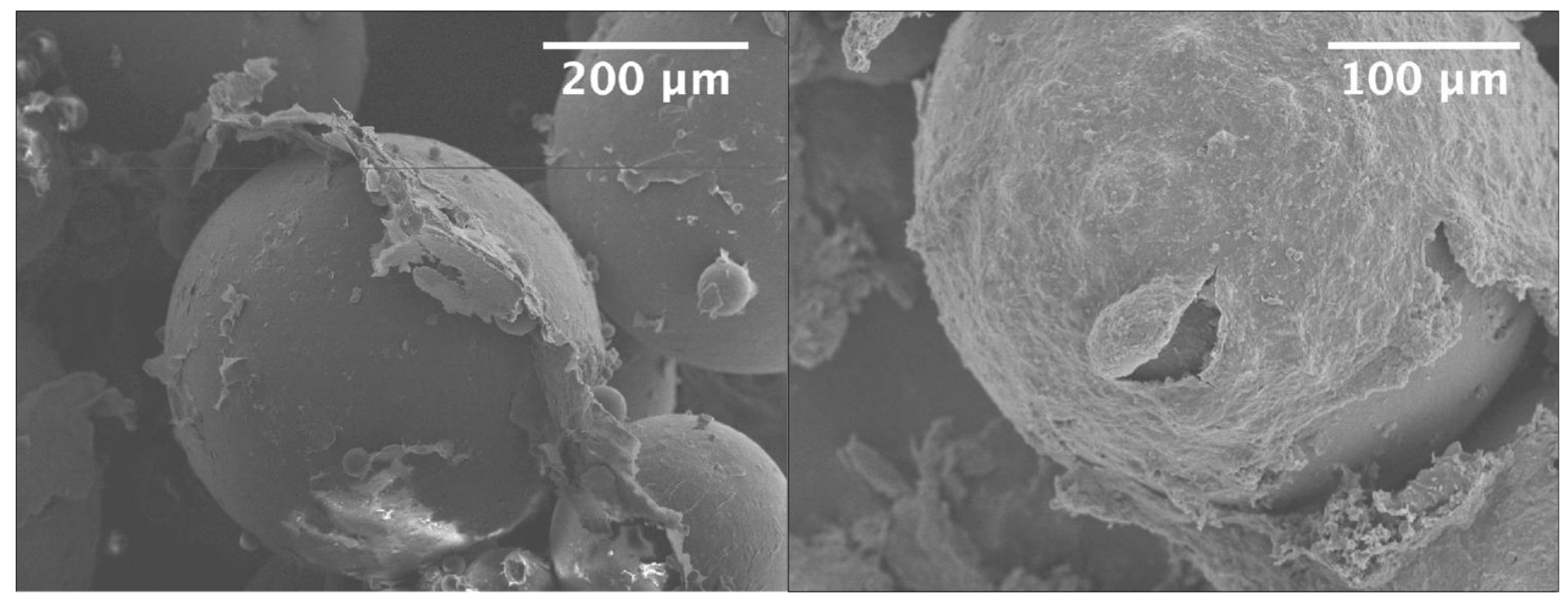

Figure 1: FESEM images of GO-coated spheres made with a 7/3 (by volume) water/oil ratio and $1 \mathrm{mg} \mathrm{GO} / \mathrm{ml}$ solution after heating to $450{ }^{\circ} \mathrm{C}$ at a rate of $20^{\circ} \mathrm{C} / \mathrm{min}$. Degrading a portion of the internal polystyrene reveals and defines the GO shell surrounding the polymer sphere.

To study the ability of GO to stabilize the water/oil interface, we change the amount of GO relative to styrene (oil), and observe the PS spheres that result. Decreasing the amount of GO relative to the amount of styrene in the emulsion results first in an increase in droplet size and then in a portion of the styrene monomer not being contained within the GO-stabilized droplets. This suggests a maximum stable droplet size, and once the maximum size is reached, the excess styrene not contained within emulsion droplets instead forms a micron to sub-micron drop size suspension in the water phase that polymerizes concurrently with the GO-coated spheres. Evidence for this can be seen in Figure 2a and Figure 2b. Both images are close ups of the surface of GO coated spheres, but the spheres in Figure 2a are made with $95 \%$ less GO than the material in Figure 2b, and the underlying spheres are roughly $250 \mu \mathrm{m}$ 
across and coated with small PS particles. In contrast, the spheres in Figure $2 \mathrm{~b}$ are roughly $50 \mu \mathrm{m}$ across and no small PS spheres are seen. Images at lower magnification showing multiple spheres are shown in Figure 2c and Figure 2d. This suggests a maximum stable sphere size, approximately $300 \mu \mathrm{m}$. Mechanistically, our system is a suspension rather than an emulsion polymerization, and monomer drops can be stabilized by GO or can be created by mixing, as is the case with the small drops seen in Figure 2 a. When the GO concentration is great enough however, the styrene monomer is contained within the GOstabilized drops, and no small PS spheres are observed, as shown in Figure $2 \mathrm{~b}$.

Our observation that increasing the concentration of $\mathrm{GO}$ in the dispersion from $0.1 \mathrm{mg} / \mathrm{mL}$ solution to $2.0 \mathrm{mg} / \mathrm{mL}$ solution (or an increase from 0.37 to $7.33 \mathrm{mg} \mathrm{GO} / \mathrm{g}$ styrene) results in significantly smaller GO coated PS spheres, as shown in in Figure 2d, is in agreement with results published by He, et al, in which increasing the concentration of GO in an oil-in-water emulsion system led to the formation of smaller oil droplets.[8] With increasing concentration of GO we find the size of the spheres reaches a minimum, approximately $30 \mu \mathrm{m}$, after which the excess GO precipitates rather than forming a thicker coating around the sphere.

In order to verify that the sizes of the GO coated PS particles correlate with the size of the emulsion droplets rather than being a result of polymerization driven rearrangement, the emulsion droplet size is measured using acoustic spectroscopy prior to polymerization. The deep black color of the emulsion absorbs light, precluding the use of light scattering techniques, but sound waves allow us to determine the size and size distribution of the droplets. Shown in Figure 2e and Figure 2f, the size of the droplets in the low concentration GO emulsion (Figure 2e) is centered around $240 \mu \mathrm{m}$, while in the high concentration GO emulsion the droplet size is centered around $90 \mu \mathrm{m}$. This closely correlates with the sizes observed in the SEM images of the particles following polymerization. To our knowledge this is the first time the global distribution of droplet sizes in a GO emulsion has been shown to correspond to the size of the templated GO coated polymer spheres. 

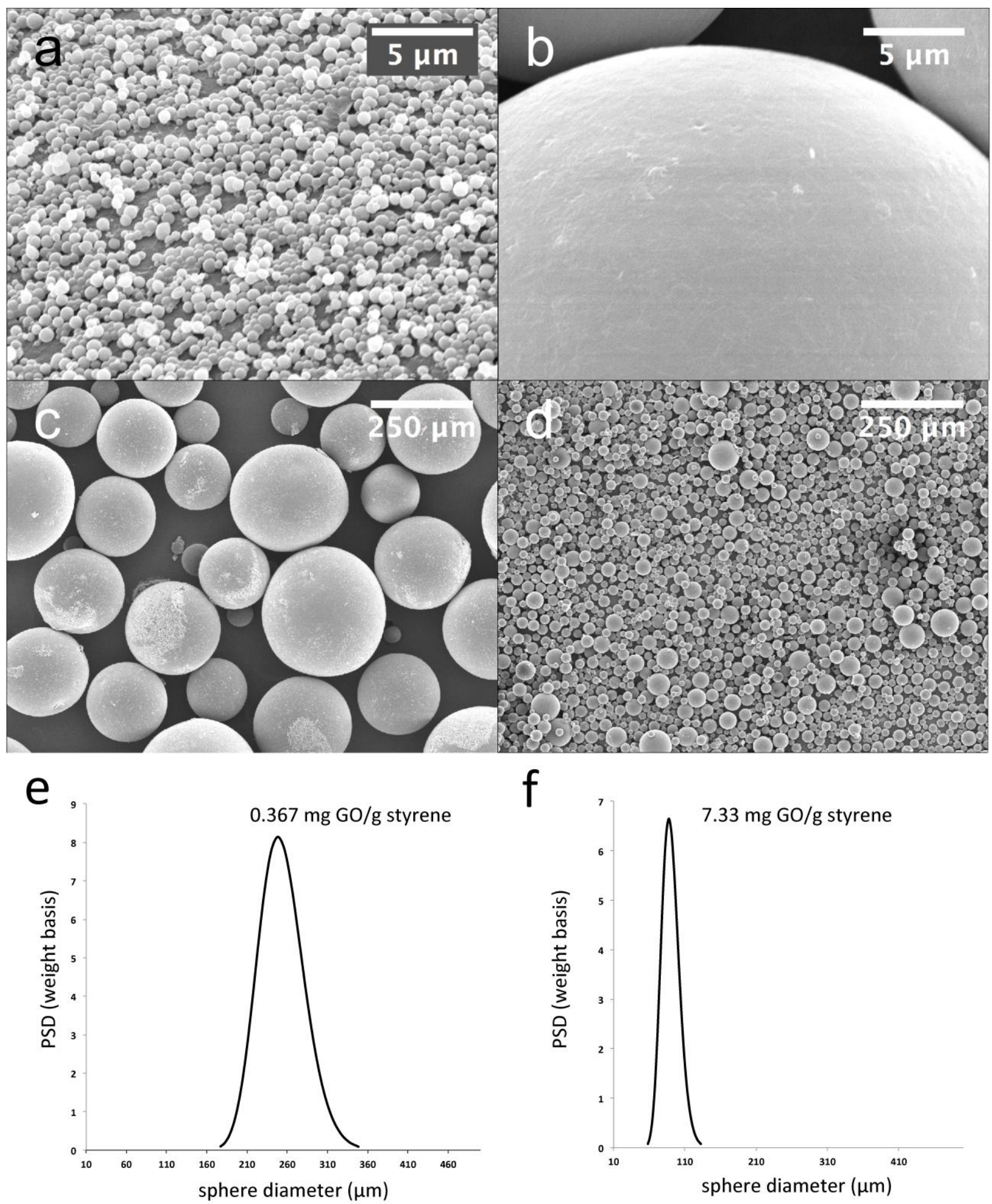

Figure 2: (a) FESEM image of large GO coated sphere covered with smaller PS spheres produced with a GO concentration of $0.367 \mathrm{mg} \mathrm{GO} / \mathrm{g}$ styrene. (b) FESEM image of surface of large sphere produced with $7.33 \mathrm{mg} \mathrm{GO} / \mathrm{g}$ styrene (note the lack of small spheres). (c) FESEM image of spheres formed with GO concentration of $0.367 \mathrm{mg}$ GO/g styrene (lower magnification compared to Fig. 2a). (d) FESEM image of spheres formed with a much higher GO concen- 
tration of $7.33 \mathrm{mg} \mathrm{GO} / \mathrm{g}$ styrene (note smaller size of GO coated spheres). (e, f) Sphere size distribution obtained by acoustic spectroscopy before polymerization of the styrene of (e) lower concentration GO emulsion and (f) higher concentration GO emulsion. The units of the vertical axis, PSD, stands for Particle Size Distribution.

Additional factors that might be expected to impact the size of the emulsion droplets include the ratio of the oil and water phases and the degree of mixing. To investigate the role of the water/oil ratio, two sets of emulsions with different water/oil ratios are examined and the results shown in Figure 3a. The GO-coated PS sphere size is plotted against the GO/styrene ratio (mg GO / g styrene) for both a 70/30 and a 30/70 styrene/water emulsion system with varying amounts of GO. All of the GO appears to reside at the water/oil interface in these systems, as no GO is observed in the water phase of the emulsion. As shown in Figure 3a, the sphere size is a function only of the GO/styrene ratio, and not the styrene/water or GO/water ratio. As the popularity of GO is largely a result of its ability to suspend in water, this result is somewhat surprising.
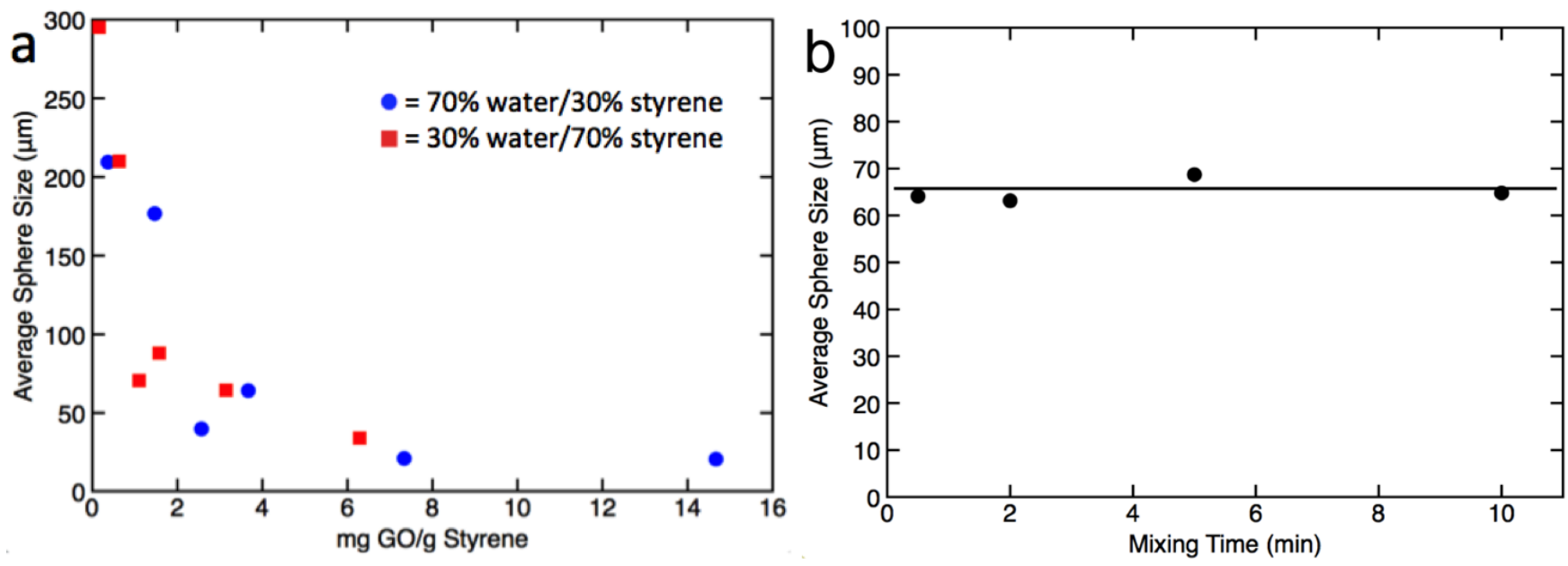

Figure 3: Factors affecting sphere size (a) Plot of sphere size as a function of GO/monomer ratio, comparing a 7/3 by volume and a $3 / 7$ by volume water/styrene mixture. Increasing the amount of GO relative to styrene is shown to decrease the sphere size in both solvent ratios. (b) Effect of emulsion blending time on the sphere size. Mixing time does not affect sphere size, leading to the conclusion that the amount of surfactant (GO) determines sphere size, rather than the emulsification process.

Previous studies have shown that the size of emulsion droplets can depend on the length of time the system is mixed.[27] In order to rule out the effect of mixing time on our observed sphere sizes, mix- 
tures with identical compositions but varying mixing times are shown to produce spheres with nearly identical sizes. A plot of sphere size as a function of mixing time is shown in Figure 3b, and shows no effect of mixing time on the size of spheres within the times investigated. In practice, all samples in our investigations are mixed for $30 \mathrm{~s}$.

Hydrophobic/Hydrophilic ratio of GO According to Bancroft's rule, the continuous phase of an emulsion is the phase in which the emulsifying particles are preferentially dispersed,[28] and we observe oil-in-water emulsions with GO in all the oil/water ratios tested. The GO is thus best described as being more hydrophilic than hydrophobic. In order to test the role of hydrophilicity and produce waterin-oil emulsions with GO, we modify the hydrophobic/hydrophilic balance of the GO sheets by functionalization with trimethylsilyl (TMS) groups. Capping oxygen functionalities on the surface of the GO sheets with TMS shifts the hydrophilic/hydrophobic balance of the GO sheets towards being more hydrophobic. Figure SI 3a shows the TGA traces of a series of silinated GO samples, showing that increasing the amount of chlorosilane reagent increases the mass of residue after high temperature treatment, suggesting an increase in Si functionalization. Based on these results, the extent of functionalization is estimated and shown in table SI 1. The presence of silane-functionalization is also confirmed by FTIR spectroscopy, shown in Figure SI 4. The gradual increase in the intensity of the Si-O-C asymmetrical stretching band at $\sim 1150 \mathrm{~cm}^{-1}$ is indicative of an increase in the TMS-functionalization in the GO sheets.[29]

Changing the hydrophobicity of the GO sheets has an immediately visible effect on the material. As illustrated in Figure 4a, when the modified GO is placed in a glass vial containing water and heptane, it climbs the walls of the vial. Woltornist, et al., showed that hydrophobic graphene sheets will also climb the walls of a vial as the graphene sheets spread at the high energy water/heptane interface.[30] The silanated GO behaves similarly to graphene, with the height of the climbing correlating with the extent of silanation. In contrast, unmodified GO shows no climbing. In addition, emulsions stabilized with sila- 
nated GO show a phase inversion, with water now dispersed in a continuous oil phase. After polymerization, a solid composite is formed. FESEM reveals that the solid composite is a porous material templated by the water-in-oil emulsion, with voids remaining after removal of the dispersed aqueous phase, show in Figure $\mathbf{4 b}$. This is in stark contrast to the powdered material formed with PS and unmodified GO, shown in Figure SI 3b.

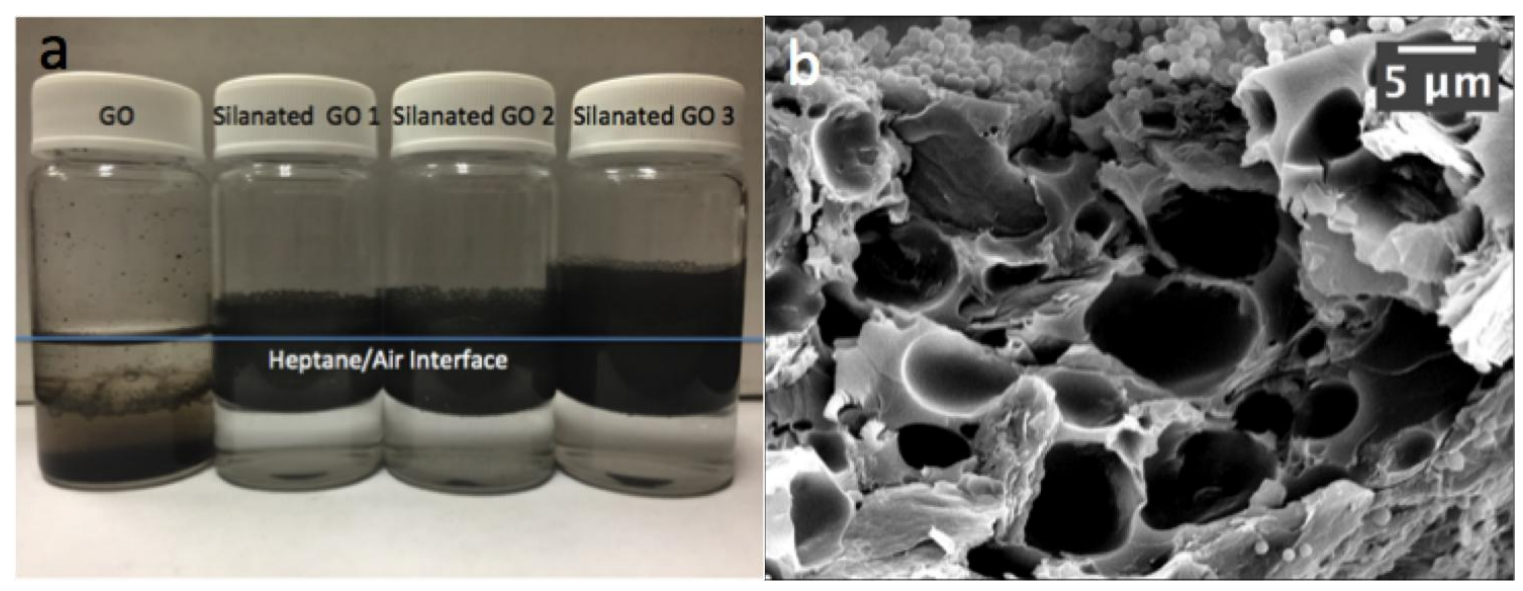

Figure 4: Silanation of GO. (a) Silanated GO climbs the wall of glass vials, stabilizing the interface between the hydrophilic glass surface and the hydrophobic heptane vapor above the solution, while unfunctionalized GO remains dispersed in the aqueous layer. GO climbs the glass vial above the heptane liquid above the line. (b) FESEM image of polystyrene composite material made with highly silanated GO. Increasing the hydrophobicity of the GO switched the emulsion formed from oil-in-water to water-in-oil, making the styrene the continuous phase and producing a foam-like composite.

Effect of Fractionation of Graphene Oxide As noted earlier, GO is not a uniformly oxidized material,[23] with some sheets more oxidized than others within the same batch. Previous work has shown, however, that GO can be separated into fractions based on the degree of oxidation of individual sheets.[24] By separating a sample of GO into more oxidized and less oxidized fractions, we use these two fractions in water/styrene emulsion systems to make GO-coated polystyrene spheres, as shown in Figure 5. As illustrated in Figure 5a, the highly oxidized GO fraction results in particles that are nearly perfectly spherical. Conversely, the less oxidized GO produces particles that are much less spherical, with flat spots and inconsistencies throughout the surface, shown in Figure 5b. 
This is due, in part, to the fact that the highly oxidized GO has more defects introduced into the original $\mathrm{sp}^{2}$-hybridized graphene lattice. By replacing the planar double bonds with single bonds, the GO sheets become less planar and more bent, as reported previously.[31] As a result, highly oxidized GO is able to accommodate the curvature of a spherical emulsion droplet in a way that the less oxidized GO cannot, although the overall size of the droplet does not significantly change. It is important to note that this less oxidized GO is not the same as reduced GO. In reduced GO, the material is first oxidized, then reduced, leaving a significant level of imperfections in the graphene lattice. In the less oxidized GO used here, a GO batch is fractioned to isolate GO sheets that contain less oxidization that than other sheets, and thus do not contain the residual imperfections from the reduction of previously oxidized material. This is the first example of non-spherical GO emulsions reported, and the first example of modifying the shape of GO coated spheres by changing the geometry of the GO through control of the degree of GO oxidation.

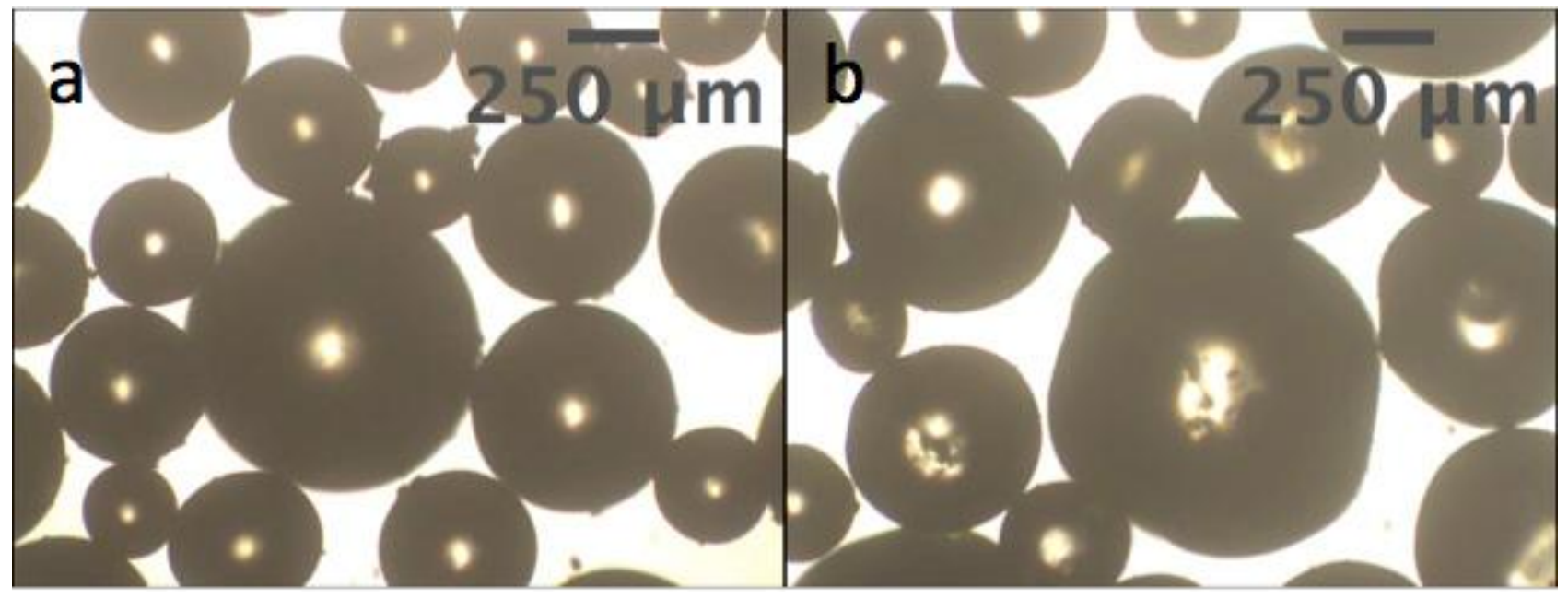

Figure 5: Effect of extent of GO oxidation on the shape of the dispersed monomer phase of the emulsion. Optical microscopy images of PS spheres produced with (a) highly oxidized GO and (b) less oxidized GO.

\section{Conclusion}

Two-dimensional surfactants such as GO are an exciting new class of materials. Their sheet-like morphology provides an advantage for stabilizing solvent interfaces, and their electrical conductivity 
promises applications not available with small molecule surfactants. Critical to their development is an understanding of the effect variations in structure and surface chemistry have on their surfactant properties. The work reported in this article begins to address these issues by varying parameters such as concentration, hydrophobicity, and sheet planarity. Variations of these parameters has been demonstrated to result in: an upper and lower limit to the size of emulsion droplets, an emulsion phase inversion with more hydrophobic sheets, and emulsion drops with irregular shapes resulting from increased planarity in less oxidized GO.

\section{Competing financial interests}

The author declares no competing financial interest.

\section{Appendix A. Supplementary data}

Supplementary data associated with this article can be found in the online version at

\section{Acknowledgements}

This work was supported by the NSF through grant number DMR1535412 and by the University of Connecticut Research Foundation.

\section{REFERENCES}

[1] B.C. Brodie, On the Atomic Weight of Graphite, Philos. Trans. R. Soc. London. 149 (1859) 249-259. doi:10.1098/rstl.1859.0013.

[2] S. Park, R.S. Ruoff, Chemical methods for the production of graphenes, Nat. Nanotechnol. 4 (2009) 217-224. doi:10.1038/nnano.2009.58.

[3] G. Yin, Z. Zheng, H. Wang, Q. Du, H. Zhang, Preparation of graphene oxide coated polystyrene microspheres by Pickering emulsion polymerization, J. Colloid Interface Sci. 394 (2013) 192-198. doi:10.1016/j.jcis.2012.11.024.

[4] P. Xie, X. Ge, B. Fang, Z. Li, Y. Liang, C. Yang, Pickering emulsion polymerization of graphene oxide-stabilized styrene, Colloid Polym. Sci. 291 (2013) 1631-1639. doi:10.1007/s00396-013-2897-x.

[5] X. Song, Y. Yang, J. Liu, H. Zhao, PS colloidal particles stabilized by graphene oxide, 
Langmuir. 27 (2011) 1186-1191. doi:10.1021/la103856h.

[6] P. Guo, H. Song, X. Chen, Hollow graphene oxide spheres self-assembled by W/O emulsion, J. Mater. Chem. 20 (2010) 4867-4874. doi:10.1039/b927302f.

[7] L.J. Cote, J. Kim, V.C. Tung, J. Luo, F. Kim, J. Huang, Graphene oxide as surfactant sheets, Pure Appl. Chem. 83 (2010) 95-110. doi:10.1351/PAC-CON-10-10-25.

[8] Y. He, F. Wu, X. Sun, R. Li, Y. Guo, C. Li, et al., Factors that Affect Pickering Emulsions Stabilized by Graphene Oxide, Appl. Mater. Interfaces. 5 (2013) 4843-4855.

[9] M.M. Gudarzi, F. Sharif, Self assembly of graphene oxide at the liquid-liquid interface: A new route to the fabrication of graphene based composites, Soft Matter. 7 (2011) 3432. doi:10.1039/c0sm01311k.

[10] J. Kim, L.J. Cote, F. Kim, W. Yuan, K.R. Shull, J. Huang, Graphene Oxide Sheets at Interfaces, J. Am. Chem. Soc. 132 (2010) 8180-8186. doi:10.1021/ja102777p.

[11] S.C. Thickett, P.B. Zetterlund, Graphene Oxide (GO) Nanosheets as Oil-in-Water Emulsion Stabilizers: Influence of Oil Phase Polarity, J. Colloid Interface Sci. 442 (2014) 67-74. doi:10.1016/j.jcis.2014.11.047.

[12] J. Sun, H. Bi, Pickering emulsion fabrication and enhanced supercapacity of graphene oxide-covered polyaniline nanoparticles, Mater. Lett. 81 (2012) 48-51. doi:10.1016/j.matlet.2012.04.078.

[13] H. Hu, X. Wang, J. Wang, L. Wan, F. Liu, H. Zheng, et al., Preparation and Properties of Graphene Nanosheets-Polystyrene Nanocomposites via in situ Emulsion Polymerization, Chem. Phys. Lett. 484 (2010) 247-253. doi:10.1016/j.cplett.2009.11.024.

[14] R. Ding, Y. Hu, Z. Gui, R. Zong, Z. Chen, W. Fan, Preparation and Characterization of Polystyrene/Graphite Oxide Nanocomposite by Emulsion Polymerization, Polym. Degrad. Stab. 81 (2003) 473-476. doi:10.1016/S0141-3910(03)00132-0.

[15] Z. Zheng, X. Zheng, H. Wang, Q. Du, Macroporous graphene oxide-polymer composite prepared through pickering high internal phase emulsions., ACS Appl. Mater. Interfaces. 5 (2013) 7974-82. doi:10.1021/am4020549.

[16] M.Z. Kassaee, E. Motamedi, M. Majdi, Magnetic Fe3O4-graphene oxide / polystyrene: Fabrication and characterization of a promising nanocomposite, Chem. Eng. J. 172 (2011) 540-549. doi:10.1016/j.cej.2011.05.093.

[17] S.H. Che Man, S.C. Thickett, M.R. Whittaker, P.B. Zetterlund, Synthesis of polystyrene nanoparticles "armoured" with nanodimensional graphene oxide sheets by miniemulsion polymerization, J. Polym. Sci. Part A Polym. Chem. 51 (2013) 47-58. doi:10.1002/pola.26341.

[18] S.C. Thickett, N. Wood, Y.H. Ng, P.B. Zetterlund, Hollow hybrid polymer-graphene oxide nanoparticles via Pickering miniemulsion polymerization., Nanoscale. 6 (2014) 8590-4. doi:10.1039/c4nr01175a.

[19] S.H. Che Man, D. Ly, M.R. Whittaker, S.C. Thickett, P.B. Zetterlund, Nano-sized graphene oxide as sole surfactant in miniemulsion polymerization for nanocomposite synthesis: Effect of pH and ionic strength, Polym. (United Kingdom). 55 (2014) 3490- 
3497. doi:10.1016/j.polymer.2014.06.005.

[20] T. Szabó, O. Berkesi, P. Forgó, K. Josepovits, Y. Sanakis, D. Petridis, et al., Evolution of surface functional groups in a series of progressively oxidized graphite oxides, Chem. Mater. 18 (2006) 2740-2749. doi:10.1021/cm060258+.

[21] A. Lerf, H. He, M. Forster, J. Klinowski, Structure of Graphite Oxide Revisited, J. Phys. Chem. B. 102 (1998) 4477-4482. doi:10.1021/jp9731821.

[22] D.C. Marcano, D. V Kosynkin, J.M. Berlin, A. Sinitskii, Z. Sun, A. Slesarev, et al., Improved Synthesis of Graphene Oxide, ACS Nano. 4 (2010) 4806-4814. doi:10.1021/nn1006368.

[23] A.M. Dimiev, J.M. Tour, Mechanism of Graphene Oxide Formation., ACS Nano. (2014). doi:10.1021/nn500606a.

[24] H. V. Kumar, S.J. Woltornist, D.H. Adamson, Fractionation and Characterization of Graphene Oxide by Oxidation Extent Through Emulsion Stabilization, Carbon N. Y. 98 (2016) 491-495.

[25] J. William S. Hummers, R.E. Offeman, Preparation of Graphitic Oxide, J. Am. Chem. Soc. 80 (1958) 1339. doi:10.1021/ja01539a017.

[26] A.S. Dukhin, P.J. Goetz, Characterization of Liquids, Nano-and Microparticulates, and Porous Bodies using Unltrasound, Elsevier, Oxford UK, 2010.

[27] G. Chen, D. Tao, An Experimental Study of Stability of Oil-Water Emulsion, Fuel Process. Technol. 86 (2005) 499-508. doi:10.1016/j.fuproc.2004.03.010.

[28] L.L. Schramm, Emulsions, Foams, and Suspensions: Fundamentals and Applications, 2006. doi:10.1002/3527606750.

[29] S. Jing, H. Lee, Chemical Bond Structure on Si-O-C Composite Films with a Low Dielectric Constant Deposited by Using Inductively Coupled Plasma Chemical Vapor Deposition, J. Korean Phys. Soc. 41 (2002) 769-773.

[30] S.J. Woltornist, A.J. Oyer, J.-M.Y. Carrillo, A. V. Dobrynin, D.H. Adamson, Conductive thin films of pristine graphene by solvent interface trapping, ACS Nano. 7 (2013) 70627066. doi:10.1021/nn402371c.

[31] D.K. Pandey, T.F. Chung, G. Prakash, R. Piner, Y.P. Chen, R. Reifenberger, Folding and cracking of graphene oxide sheets upon deposition, Surf. Sci. 605 (2011) 16691675. doi:10.1016/j.susc.2011.04.034. 